\title{
Efeitos dos alongamentos estático, balístico e facilitação neuromuscular proprioceptiva sobre variáveis de salto vertical
}

\author{
Effects of static, ballistic, and proprioceptive neuromuscular facilitation stretching \\ on vertical jump variables
}

\author{
Marcela Gomes Ferreira', Welds Rodrigo Ribeiro Bertor ${ }^{1}$, Alberito Rodrigo de Carvalho', \\ Gladson Ricardo Flor Bertolini ${ }^{1} \bowtie$ \\ ${ }^{1}$ Laboratório de Estudo das Lesões e Recursos Fisioterapêuticos da Universidade Estadual do Oeste do Paraná (Unioeste).
}

Fontes de recursos: a plataforma de salto vertical foi adquirida com fundos do Centro de Reabilitaçáo Física da Unioeste.

\section{RESUMO}

Objetivos: O alongamento muscular antes do exercício é controverso quanto aos seus efeitos em relação ao desempenho muscular. Com isso, o objetivo deste estudo foi comparar o efeito do alongamento estático, balístico e da facilitação neuromuscular proprioceptiva sobre a potência muscular, tempo e altura de salto.

Métodos: Para este estudo de intervenção, não controlado e cruzado, foram incluídos 20 jovens estudantes da Universidade Estadual do Oeste do Paraná, de ambos os sexos, com idade entre 18 a 28 anos, sem queixas de dor e sem lesões musculoesqueléticas. A amostra foi dividida em quatro subgrupos, sendo que três deles recebiam um dos três tipos de alongamento (estático, balístico ou facilitação neuromuscular proprioceptiva), enquanto o quarto subgrupo era o controle. As intervenções foram realizadas uma vez por semana, na forma de rodízio, ao longo de quatro semanas, ao final das quais todos os subgrupos haviam realizado todos os procedimentos em estudo. Logo após os alongamentos (ou isoladamente, nos controles) eram realizados saltos em uma plataforma de saltos verticais e avaliadas variáveis relativas ao desempenho nessa prova. A análise estatística foi feita por meio da Análise de Variância com medidas repetidas, com nível de significância de 5\%.

Resultados: Analisando-se as médias obtidas pelos quatro grupos para as variáveis do salto vertical, não houve diferença significativa na potência muscular, tempo ou altura de salto, antes e após a aplicação dos três tipos de alongamento. Os valores de p obtidos foram 0,508 para potência muscular; 0,674 para tempo de salto; e 0,606 para altura de salto.

Conclusões: Se há objetivo de ganho de extensibilidade prévio à atividade física que envolva salto e potência, não se contraindica o uso de alongamento, visto que parece não haver influência negativa deste sobre variáveis importantes para o salto em jovens saudáveis, pelo menos dentro de um período de quatro semanas.

DESCRITORES: força muscular; exercícios de alongamento muscular; terapia por exercício.

\section{ABSTRACT}

Aims: The effects of muscle stretching before exercise on muscle performance is controversial. The aims of this study were to compare the effects of static, ballistic, and proprioceptive neuromuscular facilitation (PNF) stretching on muscle power, flight time, and jump height.

Methods: This uncontrolled, crossed intervention study included 20 young male and female students aged 18 to 28 years from Universidade Estadual do Oeste do Paraná, without any complaints of pain and with no musculoskeletal injuries. The sample was divided into four subgroups: three of them were submitted to static, ballistic, and PNF stretching, while one was the control group. The interventions occurred once weekly, alternating between the stretching modalities, over a 4-week period, at the end of which all subgroups had performed all the exercises proposed. Vertical jumps were performed on a testing mat immediately after the stretching exercises (or separately in the case of controls) in order to assess muscle performance. The repeated measures ANOVA was used for the statistical analysis and the significance level was set at $5 \%$.

Results: There was no significant difference in muscle power, flight time, or jump height before and after the application of the three stretching modalities. The following $\mathrm{p}$ values were obtained: 0.508 for muscle power, 0.674 for flight time, and 0.606 for jump height.

Conclusions: Stretching is not contraindicated if the goal is to enhance muscle extensibility prior to vertical jumps, since, apparently, stretching has no negative effect on important variables among healthy young individuals, at least not within a 4-week period.

KEY WORDS: muscle strength; muscle stretching exercises; exercise therapy. 
Abreviaturas: FNP, facilitação neuromuscular proprioceptiva.

\section{INTRODUÇÃO}

A extensibilidade muscular é considerada uma qualidade física importante no aprimoramento da aptidão física, reabilitação de lesões e desempenho esportivo [1], sendo que os exercícios de alongamento são técnicas utilizadas para aumentar a extensibilidade do tecido conjuntivo muscular e periarticular [2].

O encurtamento é caracterizado pela perda da extensibilidade dos tecidos moles, ou seja, redução parcial do comprimento de uma unidade musculotendínea, resultando em limitação na mobilidade articular [3]. O alongamento produz efeitos imediatos, e uma única sessão, em indivíduos saudáveis, é capaz de melhorar a extensibilidade muscular e a mobilidade articular [4,5].

$\mathrm{O}$ alongamento muscular antes da prática esportiva apresenta controvérsias em relação aos seus benefícios, no que diz respeito ao desempenho muscular [6]. É prática comum recomendar-se aos atletas a realização de alongamento estático, antes de atividades esportivas, contudo sem conhecimento sobre a real repercussão sobre o desempenho esportivo. Alguns estudos sugerem, inclusive, que o alongamento prévio à prática desportiva pode causar diminuição no pico de potência e de torque. Estes fatos justificam a necessidade de mais pesquisas na área [7].

Dentre os diferentes tipos de alongamento utilizados antes da atividade esportiva, pode-se citar o estático, o balístico e modalidades que utilizam facilitação neuromuscular proprioceptiva (FNP) [8]. O alongamento estático é uma técnica de tensão isométrica aplicada lentamente a um músculo na sua maior extensão e mantida em tal posição por um período de tempo [9]. No alongamento balístico o músculo é submetido a um estiramento até o final da amplitude de movimento por uma força externa ou pela musculatura antagonista ao movimento; uma vez alcançada a máxima amplitude, utilizam-se contrações musculares insistidas do tipo "sacudidas", buscando maior alcance de movimento [10,11]. A FNP é um método que favorece o mecanismo neuromuscular mediante a estimulação dos proprioceptores. Utiliza-se de contração muscular isométrica voluntária máxima seguida pelo relaxamento $[10,12]$.

Existem controvérsias sobre a possibilidade de o alongamento estático antes do exercício produzir um efeito negativo sobre o desempenho muscular máximo, e poucos estudos investigaram os efeitos agudos do alongamento balístico e da FNP neste desempenho. [13]. O máximo desempenho em salto vertical é imprescindível para o sucesso de atletas em diversas modalidades esportivas [14], e também poucos estudos analisaram se o alongamento influencia na altura e no tempo de salto. Tendo em vista estas dúvidas sobre os efeitos dos diferentes tipos de alongamento realizados antes de atividades físicas, o objetivo do presente estudo foi comparar o efeito imediato do alongamento estático, balístico e da FNP sobre a potência muscular, tempo e altura de salto em indivíduos saudáveis.

\section{MÉTODOS}

O presente estudo de intervenção, não controlado e cruzado, foi aprovado pelo Comitê de Ética em Pesquisa com Seres Humanos da Universidade Estadual do Oeste do Paraná (Unioeste), sob parecer 047/2013-CEP. O estudo foi realizado durante o segundo semestre do ano de 2013. A amostra foi escolhida por conveniência e a participação foi de forma voluntária, sendo composta por 20 jovens estudantes da Unioeste, de ambos os sexos, sendo que para tal tamanho de amostra, com vistas na variável potência, com desvio-padrão de 70,0, para diferença a ser detectada de 55,0, com nível de significância de $5 \%$, o poder do teste foi de $80 \%$ [15].

Foram incluídos na pesquisa indivíduos com idade entre 20 e 25 anos, sem lesões musculoesqueléticas ou história de cirurgia na coluna, sem queixas de dor e sem relato de tonturas e/ou vertigens, que se comprometeram a não alterar sua rotina de atividades físicas durante $o$ período de avaliação. No encontro inicial foi explicado o procedimento e solicitada a assinatura do termo de consentimento livre e esclarecido.

As variáveis foram analisadas por meio de saltos verticais na plataforma Jump Test (Hidrofit Ltda., Belo Horizonte, MG). A potência, tempo e altura dos saltos, antes e depois dos alongamentos, foram calculados pelo software Multisprint, conectado à plataforma.

Foram estudadas três formas de alongamento: alongamento estático, balístico e FNP. Cada voluntário realizou todas as formas de alongamento, uma a cada avaliação, sendo seu próprio controle no final. Para tanto, a amostra foi dividida em quatro subgrupos (A, B, C e D), com cinco voluntários em cada. Em cada etapa, três subgrupos recebiam um dos três tipos de alongamento, e o quarto subgrupo era o controle. As intervenções foram realizadas uma vez por semana, na forma de rodízio, ao longo de quatro semanas, em que cada voluntário compareceu ao Centro de Reabilitação Física da Unioeste (Tabela 1). 
Tabela 1. Demonstração da sequência de intervenção de acordo com cada grupo, conforme a semana.

\begin{tabular}{ccccc}
\hline \multirow{2}{*}{ Semana } & \multicolumn{4}{c}{ Tipo de intervenção } \\
\cline { 2 - 5 } & Controle & Balístico & Estático & FNP \\
\hline 1 & A & B & C & D \\
2 & D & C & A & B \\
3 & B & A & D & C \\
4 & C & D & B & A \\
\hline
\end{tabular}

FNP: facilitação neuromuscular proprioceptiva.

Cada participante realizou uma pesagem, em balança digital (Indústrias Filizola, Porto Alegre, RS), no início do experimento. O peso foi utilizado no programa Multisprint para o cálculo dos resultados.

Inicialmente realizavam-se três saltos verticais, com contramovimento, com as mãos apoiadas na cintura, sem auxílio dos membros superiores e sem pausa, na plataforma. Em seguida, era aplicada uma das técnicas de alongamento no grupo muscular tríceps sural (ou repouso, para o grupo controle) em ambos os membros.

As três técnicas de alongamento selecionadas foram realizadas com o voluntário em decúbito dorsal sobre uma maca. A técnica de alongamento estática ocorreu com dorsiflexão voluntária máxima de tornozelo, o terapeuta mantendo a posição com auxílio das mãos durante 30 segundos. A técnica de alongamento balístico foi realizada com dorsiflexão voluntária máxima de tornozelo, o terapeuta realizando movimentos rítmicos (frequência de 30 movimentos por minuto), com velocidade progressiva, durante 30 segundos, no sentido do alongamento, até chegar à máxima amplitude. Para a FNP foi realizada a dorsiflexão voluntária máxima de tornozelo, o terapeuta oferecendo resistência à contração isométrica do tríceps sural, enquanto o voluntário tentava realizar flexão plantar, contraindo durante 6 segundos, com posterior relaxamento de 4 segundos, repetindo o procedimento três vezes, com período de alongamento total de 18 segundos e relaxamento de 12 segundos. Todos os alongamentos foram realizados por um mesmo terapeuta.
Após a aplicação do alongamento, aguardava-se 30 segundos e realizava-se outros três saltos verticais sem pausa. Por meio dos saltos, o software Multisprint calculava os tempos de voo (em segundos), os contatos com a plataforma e os tempos totais entre os dois saltos (que é o tempo de salto completo), a potência muscular (em watts) e a altura do salto (em centímetros).

Para avaliar o possível efeito de cada tipo de alongamento sobre a força muscular, os dados obtidos em cada um dos quatro grupos em cada semana do experimento foram reunidos conforme cada variável do salto vertical (altura, potência e tempo de salto), e calculadas as suas médias e respectivos desvios padrões. Com o objetivo de verificar se existiu diferença significativa entre as médias das amostras antes e depois das atividades de alongamento (balístico, estático, FNP e controle) realizou-se a Análise de Variância com medidas repetidas, com nível de significância de 5\%, com auxílio do programa IBM SPSS 18.0.

\section{RESULTADOS}

Foram estudados 20 indivíduos, sendo 15 do sexo feminino. Doze eram sedentários, sete faziam musculação e um praticava esporte três vezes na semana. Os dados antropométricos da amostra estão descritos na Tabela 2.

Tabela 2. Apresentação dos dados antropométricos dos voluntários da pesquisa, de acordo com o gênero.

\begin{tabular}{ccccc}
\hline Gênero & Idade & Altura & Peso & IMC \\
Feminino $(n=15)$ & $22 \pm 0,53$ & $1,64 \pm 0,05$ & $58 \pm 5,6$ & $20 \pm 2,3$ \\
Masculino $(n=5)$ & $23 \pm 1,9$ & $1,77 \pm 0,07$ & $68 \pm 1,3$ & $21 \pm 1,5$ \\
\hline
\end{tabular}

IMC: índice de massa corporal.

Os resultados encontrados para as três variáveis estudadas, considerando-se as médias dos quatro grupos, antes e após as intervenções de alongamento, não mostraram diferenças significativas entre cada intervenção e nem em relação ao grupo controle (Tabela 3).

Tabela 3. Valores médios (considerando a média dos quatro grupos estudados) obtidos para cada variável do salto vertical (potência, tempo e altura), tanto pré quanto pós-intervenção, conforme o tipo de intervenção realizada (alongamento balístico, estático e facilitação neuromuscular proprioceptiva) e controles (sem intervenção).

\begin{tabular}{|c|c|c|c|c|c|c|}
\hline \multirow{2}{*}{\multicolumn{2}{|c|}{ Variáveis do salto vertical }} & \multirow{3}{*}{$\begin{array}{c}\begin{array}{c}\text { Controle } \\
\text { média } \pm \text { DP }\end{array} \\
290,8 \pm 73,5\end{array}$} & \multicolumn{3}{|c|}{ Tipo de alongamento } & \multirow[b]{2}{*}{$\begin{array}{l}\text { Estatística } \mathrm{F}^{*} \\
\mathrm{p}^{*}\end{array}$} \\
\hline & & & \multirow{2}{*}{$\begin{array}{c}\text { Balístico } \\
\text { média } \pm \text { DP } \\
298,7 \pm 80,3\end{array}$} & \multirow{2}{*}{$\begin{array}{c}\begin{array}{c}\text { Estático } \\
\text { média } \pm \text { DP }\end{array} \\
301,1 \pm 84,5\end{array}$} & \multirow{2}{*}{$\begin{array}{c}\text { FNP } \\
\text { média } \pm \text { DP } \\
303,7 \pm 72,3\end{array}$} & \\
\hline Potência & Pré & & & & & $F(7,133)=0,901$ \\
\hline (watts) & Pós & $295,8 \pm 74,9$ & $299,6 \pm 84,1$ & $303,4 \pm 82,1$ & $305,9 \pm 76,8$ & $p=0,508$ \\
\hline \multirow{2}{*}{$\begin{array}{l}\text { Tempo } \\
\text { (segundos) }\end{array}$} & Pré & $2,47 \pm 2,14$ & $2,11 \pm 1,11$ & $1,89 \pm 1,37$ & $2,58 \pm 3,01$ & $F(7,133)=0,698$ \\
\hline & Pós & $2,37 \pm 1,73$ & $2,10 \pm 1,05$ & $2,40 \pm 0,96$ & $1,75 \pm 0,86$ & $p=0,674$ \\
\hline \multirow{2}{*}{$\begin{array}{l}\text { Altura } \\
\text { (centímetros) }\end{array}$} & Pré & $19,11 \pm 7,35$ & $19,93 \pm 7,79$ & $20,28 \pm 8,02$ & $20,54 \pm 7,31$ & $F(7,133)=0,779$ \\
\hline & Pós & $19,75 \pm 7,49$ & $20,45 \pm 9,20$ & $20,58 \pm 7,95$ & $20,90 \pm 8,18$ & $p=0,606$ \\
\hline
\end{tabular}

DP: desvio padrão; FNP: facilitação neuromuscular proprioceptiva. * ANOVA (análise de variância). 
A ausência de diferenças ocorreu tanto na comparação entre os grupos, quanto na comparação antes a após alongamento dentro do mesmo grupo $(\mathrm{p}>0,05)$.

\section{DISCUSSÃO}

$\mathrm{Na}$ presente pesquisa as médias das variáveis relacionadas ao salto vertical (altura, potência e tempo) não apresentaram diferenças entre si considerando os valores antes a após os tipos de alongamento (estático, balístico e FNP), e também não houve diferença em relação ao grupo controle. Resultados similares foram encontrados por Bergamin et al. [16], que compararam os efeitos do aquecimento com alongamentos estáticos e saltos versus aquecimento padrão sobre o desempenho da impulsão vertical, em atletas infantis de futebol. Realizaram avaliações em duas semanas, durante o período de competição, sendo que observaram resultados similares para os dois protocolos, sem alteração no desempenho da impulsão vertical.

No estudo de Ferreira et al. [17] foi avaliado o efeito agudo dos exercícios de alongamento estático e alongamento dinâmico, na impulsão vertical e na amplitude do movimento, em jogadores de futebol profissionais. Diferentemente do estudo anterior, os autores verificaram que o alongamento dinâmico apresentou uma média de impulsão vertical maior que a do estático. Houve discordância em relação ao alongamento dinâmico apresentar-se mais eficiente que o estático, mas houve concordância em relação ao alongamento não apresentar diferenças para o controle [17].

No presente estudo não houve diminuição na potência, tempo e altura de salto após os alongamentos. O estudo de Nogueira et al. [18], com 12 adultos do sexo masculino, mostrou redução significativa na carga máxima alcançada em membros superiores, após sessões longas (maiores que oito minutos) de alongamento. Já no estudo de Galdino et al. [19], que teve por objetivo verificar uma possível queda dos níveis de força explosiva de membros inferiores após a realização de uma rotina de exercícios de alongamento passivo, foram selecionados 21 voluntários que fizeram um aquecimento em um ciclo ergômetro estacionário durante cinco minutos e logo após executaram um teste de salto vertical. Posteriormente, foi realizado alongamento passivo nos membros inferiores dos voluntários, e em seguida novo salto; tal protocolo foi repetido a cada 10 minutos, até 30 minutos após o segundo salto. Houve uma diminuição significativa no valor médio entre o salto antes e após o alongamento. Os autores concluíram que a realização de exercícios de alongamento passivo antes de atividades que envolvam força explosiva de membros inferiores diminui o rendimento. Ambas as pesquisas apresentam discordância com o presente estudo quanto à redução do rendimento em atividades que envolvam potência e salto.

Os tempos de alongamento utilizados na atual pesquisa foram de 30 segundos para todos os alongamentos. Conceição et al. [20] verificaram diferentes tempos de alongamento estático $(10,20,40$ e 60 segundos), visando determinar o tempo mínimo de maior eficiência para o ganho da flexibilidade em voluntários jovens, em oito semanas de treinamento. Relatam que todos os grupos obtiveram ganhos significativos de flexibilidade, contudo, sem diferenças quando comparados entre si. Sendo assim, 30 segundos foi considerado um tempo efetivo para o alongamento.

Quanto ao volume de alongamento, foi aplicada apenas uma série no presente estudo, sendo que Nodari et al. [21], que avaliaram a aplicação de diferentes volumes de alongamento estático sobre a força explosiva em 24 mulheres adultas, mostraram que não houve diferença significativa na força explosiva após três dias de testes. Concluíram, assim, que diferentes volumes de alongamento estático não causam reduções proporcionais na força explosiva.

Deve-se levar em consideração, como limitação do presente estudo, que não foram analisados os efeitos crônicos dos alongamentos sobre as variáveis. Sugere-se que sejam realizadas novas pesquisas avaliando também o efeito crônico do alongamento. Conclui-se que as diferentes formas de alongamento não exerceram influência sobre a potência muscular, altura e tempo de salto, em jovens saudáveis. Se há objetivo de ganho de extensibilidade prévio à atividade física que envolva salto e potência, não se contraindica o uso de alongamento, visto que parece não haver influência negativa deste sobre variáveis importantes para o salto, pelo menos dentro de um período de quatro semanas. 


\section{REFERÊNCIAS}

1. Voigt L, Vale RGDS, Novaes J da S, Lima J, Dantas EHM. Efeito de uma e três repetições de 10 segundos de insistência do método estático para o aumento da flexibilidade em homens adultos jovens. Acta Sci Heal Sci. 2011;33(1):59-64. http://dx.doi.org/10.4025/actascihealthsci. v33i1.7896

2. Almeida PHF de, Barandalize D, Ribas DIR, Gallon D, Macedo ACB de, Gomes ARS. Alongamento muscular: suas implicações na performance e na prevenção de lesões / Muscle stretching: implications at the performance and injury prevention. Fisioter Bras. 2009;22(3):335-43.

3. Tirloni AT, Belchior ACG, Carvalho PDTC De, Reis FA dos. Efeito de diferentes tempos de alongamento na flexibilidade da musculatura posterior da coxa/Effect of different stretching durations on posterior thigh muscle flexibility. Fisioter e Pesqui. 2008;15(1):47-52.

4. Katalinic OM, Harvey LA, Herbert RD, Moseley AM, Lannin NA, Schurr K. Stretch for the treatment and prevention of contractures. Cochrane Database Syst Rev [Internet]. 2010 Jan [cited 2013 May 22];(9):CD007455. Available from: http://www.ncbi.nlm.nih.gov/ pubmed/20824861

5. Harvey L, Herbert R, Crosbie J. Does stretching induce lasting increases in joint ROM? A systematic review. Physiother Res Int. 2002;7(1):1-13. http://dx.doi.org/10.1002/pri.236

6. Ramos GV, Santos RR dos, Gonçalves A. Influência do alongamento sobre a força muscular: uma breve revisão sobre as possíveis causas /The effect of stretching on muscle strength: a short review of possible causes. Rev Bras Cineantropom Desempenho Hum. 2007;9(2): 203-6.

7. Alencar TAM Di, Matias KF de S. Princípios fisiológicos do aquecimento e alongamento muscular na atividade esportiva. Rev Bras Med Esporte. 2010;16(3):230-4. http://dx.doi.org/10.1590/S1517-86922010000300015

8. Gama ZA da S, Medeiros CADS, Dantas AVR, Souza TO de. Influência da freqüência de alongamento utilizando facilitação neuromuscular proprioceptiva na flexibilidade dos músculos isquiotibiais. Rev Bras Med Esporte. 2007;13(1):33-8. http://dx.doi.org/10.1590/s151786922007000100008

9. Lima RCM, Pessoa BF, Martins BLT, Freitas DBN de. Analysis of durability of hamstring stretching effect in two forms of intervention. Acta Fisiatr. 2006;13(1):32-8.

10. Ayala F, Baranda PS de, Cejudo A. El entrenamiento de la flexibilidad: técnicas de estiramiento. Rev Andal Med Deport. 2013;5(3): 105-12. http://dx.doi.org/10.1016/S1888-7546(12)70016-3

11. Badaro AFV, Silva AH, Beche D. Flexibilidade versus alongamento: esclarecendo as diferenças/Flexibility and stretching: review of concepts and applicability. Saúde. 2007;33(1):32-6.

12. Feland JB, Marin HN. Effect of submaximal contraction intensity in contract-relax proprioceptive neuromuscular facilitation stretching. Br J Sport Med. 2004;38(4):18-9. http://dx.doi.org/10.1136/bjsm.2003.010967

13. Bradley PS, Olsen PD, Portas MD. The effect of static, ballistic, and proprioceptive neuromuscular facilitation stretching on vertical jump performance. J Strength Cond Res. 2007;21(1):223-6. http://dx.doi.org/10.1519/00124278-200702000-00040

14. Gomes MM, Pereira G, Freitas PB De, Barela JA. Características cinemáticas e cinéticas do salto vertical: comparação entre jogadores de futebol e basquetebol / Kinematic and kinetic characteristics of vertical jump: comparison between soccer and basketball players. Rev Bras Cineantropom Desempenho Hum. 2009;11(4):392-9.

15. Lee. Tamanho de amostra para pesquisa em ciências da saúde. [Internet]. 2013. Available from: http://www.lee.dante.br/pesquisa/ amostragem/calculo_amostra.html

16. Bergamin LF, Coledam DHC, Talamoni GA, Santos JW dos. Efeitos dos aquecimentos com alongamentos ou com saltos sobre a impulsão vertical em jogadores infantis de futebol. Coleção Pesqui Educ Física. 2009;8(5):175-80.

17. Ferreira VDS, Muller BC, Achour Junior A. Efeito agudo de exercícios de alongamento estático e dinâmico na impulsão vertical de jogadores de futebol. Motriz. 2013;19(2):450-9. http://dx.doi.org/10.1590/s1980-65742013000200022

18. Nogueira CJ, Galdino LA dos S, Vale RG de S, Dantas EHM. Efeito agudo do alongamento submáximo e do método de Facilitação Neuromuscular Proprioceptiva sobre a força explosiva. HU Rev. 2009;35(1):43-8.

19. Galdino LA dos S, Nogueira CJ, César EP, Fortes MEP, Perrout JR, Dantas EHM. Potência após flexionamento. Fit Perf J. 2005;4(1):11-5.

20. Conceição MC de SC, Vale RG de S, Bottaro M, Dantas EHM, Novaes J da S. Efeitos de quatro tempos diferentes de permanência de flexionamento estático na flexibilidade de adultos jovens. Fit Perf J. 2008;7(2):88-92. http://dx.doi.org/10.3900/fpj.7.2.88.p

21. Nodari Junior RJ, Galdino LAS, Nogueira CJ, Dantas EHM. Comparação entre diferentes volumes de flexionamento sobre a força explosiva /Comparison between different volumes of flexibilising upon explosive strength. R Bras Ci e Mov. 2012;20(3):72-8. 